\title{
Volumetric Assessment of Optic Nerve Sheath and Hypophysis in Idiopathic Intracranial Hypertension
}

\author{
J. Hoffmann, C. Schmidt, H. Kunte, R. Klingebiel, L. Harms, H.-J. Huppertz, L. Lüdemann, and E. Wiener
}

\begin{abstract}
BACKGROUND AND PURPOSE: Idiopathic intracranial hypertension is a headache syndrome characterized by increased CSF pressure. Compression of the hypophysis and distension of the optic nerve sheath are reliable imaging signs. The purpose of the study was to validate, in patients with idiopathic intracranial hypertension, MR imaging-based volumetric measurements of the optic nerve sheath and hypophysis as an objective observation method for more accurate diagnosis and posttreatment follow-up.
\end{abstract}

MATERIALS AND METHODS: Twenty-three patients with idiopathic intracranial hypertension as well as age-, sex-, and body mass index-matched controls underwent volumetric measurements of the optic nerve, optic nerve sheath, and hypophysis on high-resolution T2-weighted MR images by using a 7-cm surface coil, followed by correlation with CSF opening pressures and clinical symptom scores of visual disturbances and headache.

RESULTS: Mean values of optic nerve sheath $\left(341.86 \pm 163.69 \mathrm{~mm}^{3}\right.$ versus $\left.127.56 \pm 53.17 \mathrm{~mm}^{3}, P<.001\right)$ and hypophysis volumes ( $554.59 \pm$ $142.82 \mathrm{~mm}^{3}$ versus $686.60 \pm 137.84 \mathrm{~mm}^{3}, P<.05$ ) differed significantly between healthy and diseased subjects. No significant differences between mean optic nerve volumes were observed. Receiver operating characteristic analysis showed optic nerve sheath volumes of $>201.30 \mathrm{~mm}^{3}$ (sensitivity, $86.96 \%$; specificity, $91.30 \%$ ) and hypophysis volumes of $<611.21 \mathrm{~mm}^{3}$ (sensitivity, $78.26 \%$; specificity, $69.57 \%$ ) to be indicative of idiopathic intracranial hypertension diagnosis. In patients with idiopathic intracranial hypertension, no correlations were found between optic nerve sheath and hypophysis volumes and CSF opening pressures or clinical scores of visual disturbances and headache.

CONCLUSIONS: Semiautomated volumetric measurement of optic nerve sheath and hypophysis has the potential to more accurately diagnose and follow patients with idiopathic intracranial hypertension.

ABBREVIATIONS: $c c=$ correlation coefficient; $I I H=$ idiopathic intracranial hypertension; $\mathrm{ON}=$ optic nerve; ONS = optic nerve sheath

$l^{2}$ diopathic intracranial hypertension (IIH) is an uncommon headache syndrome associated with elevated intracranial pressure in the absence of a space-occupying lesion. Initially used terms such as "meningitis serosa" coined by Quincke in 1893 or "pseudotumor cerebri" by Nonne in 1904 are now obsolete and

\footnotetext{
Received April 9, 2013; accepted after revision June 4.

From the Departments of Neurology (J.H., H.K., L.H.) and Neuroradiology (C.S., R.K., E.W.), Charité-Universitätsmedizin Berlin, Berlin, Germany; Klinik im Park (R.K.), Institute of Neuroradiology and Radiology, Zürich, Switzerland; Medical Imaging (H.-J.H.), Swiss Epilepsy Centre, Zürich, Switzerland; and Department of Radiotherapy (L.L.), University Clinic Essen, Essen, Germany.

J. Hoffmann and C. Schmidt contributed equally to this work.

Please address correspondence to Edzard Wiener, MD, Department of Neuroradiology, Charité-Universitätsmedizin Berlin, Charitéplatz 1, 10117 Berlin, Germany; e-mail: Edzard.Wiener@charite.de

$\equiv$ Indicates article with supplemental on-line table

Indicates article with supplemental on-line figure

http://dx.doi.org/10.3174/ajnr.A3694
}

have been replaced in recent classifications by the descriptive denomination "idiopathic intracranial hypertension." 1,2 Because IIH predominantly affects obese women of childbearing age and the incidence of obesity in industrialized countries is growing, $\mathrm{IIH}$ has been the focus of recent research. The underlying pathophysiologic mechanism of the disease is not fully understood. Diagnosis of IIH is based on clinical symptoms such as headache and visual disturbances; characteristic neuroimaging findings, ${ }^{3}$ including a compression of the hypophysis (ie, empty sella); flattening of the posterior globe; and a dilated optic nerve sheath (ONS) in combination with an opening pressure during lumbar puncture of $>25 \mathrm{~cm} \mathrm{H}_{2} \mathrm{O}$, with normal CSF parameters (modified Dandy criteria). ${ }^{4-6}$ The diagnostic criteria have undergone several modifications, and the disease is now defined in the Headache Classification of the International Headache Society. ${ }^{7}$ The interpretation of subtle changes on MR images obtained for the initial diagnosis or for follow-up after treatment can be challenging. 
We compared hypophysis and optic nerve sheath and optic nerve $(\mathrm{ON})$ volumes measured on high-resolution MR images in patients with IIH and age-, sex-, and body mass index-matched controls to evaluate the potential of MR imaging-based volumetric measurements as an objective method to diagnose $\mathrm{IIH}$ and follow-up effects of treatment.

\section{MATERIALS AND METHODS}

The study was approved by the local ethics committee of the Charite-Universitätsmedizin Berlin. A statement of written informed consent was obtained from all patients and healthy volunteers participating in the study.

\section{Patient Subjects}

Clinical records of 190 patients with IIH referred to our clinic between November 2005 and May 2010 were retrospectively screened. We therefore searched the electronic medical records system of the university hospital via retrieval algorithms for the International Classification of Diseases-10 classification code G93.2. ${ }^{8}$ We enrolled only patients with a minimum age of 18 years at presentation and a definite diagnosis of IIH according to the revised Dandy criteria: 1) signs and symptoms of increased intracranial pressure (headache, nausea, vomiting, transient visual obscurations, or papilledema); 2) no focal signs except abducens nerve palsy; 3) CSF opening pressure of $>25 \mathrm{~cm} \mathrm{H}_{2} \mathrm{O}$ with normal CSF composition; and 4) evidence of neither hydrocephalus, mass, structural, or vascular lesion on MR imaging nor any other cause of intracranial hypertension identified. ${ }^{9}$ All patients were of white descent. Exclusion criteria comprised systemic conditions or medication that might have an effect on intracranial pressure, inadequate follow-up, pregnancy or postpartum status, body weight $>160 \mathrm{~kg}$, and clinical history of surgical procedures affecting CSF circulation (eg, shunting procedures or fenestration of the ONS). Medical histories of 190 patients were analyzed to identify those who fully met the inclusion criteria. Because IIH is often regarded as a diagnosis of exclusion, many of the retrospectively identified patients had a probable IIH but did not fulfill all the required criteria for the diagnosis and could, therefore, not be included in the study. Other patients had additional diseases and were therefore excluded from study participation. We found only 71 patients who definitely met the modified Dandy criteria. Fifteen patients were excluded by the exclusion criteria: shunt surgery $(n=6)$, body weight $>160 \mathrm{~kg}(n=3)$, pregnancy $(n=1)$, and claustrophobia $(n=4)$. One patient $(n=1)$ fulfilled the criteria for major depression (diagnosed by the Becks Depression Inventory and Hamilton Rating Scale for Depression) and was ruled out. Eighteen patients could not be reached; 15 refused participation in the study.

The mean age of the remaining 23 patients ( 20 women, 3 men) was $37.04 \pm 13.72$ years (range, $20-63$ years). The mean body mass index was $33.51 \pm 7.67 \mathrm{~kg} / \mathrm{m}^{2}$ (range, $24.9-54.57 \mathrm{~kg} / \mathrm{m}^{2}$ ). All patients were carefully questioned about disease symptoms by using a standardized clinical questionnaire (based on the modified Dandy criteria and the diagnostic criteria established by the International Headache Society). During hospitalization, all patients with IIH underwent lumbar puncture, and CSF opening pressures were obtained before MR imaging.
Measurements of visual acuity and perimetry of the visual field were performed.

\section{Control Subjects}

Twenty-three healthy controls of white descent were included by using a match-to-pair technique regarding sex $(n=20$ women, $n=3$ men, $P=1.0$ ), age (mean, $37.91 \pm 11.84$ years [range, 22-61 years], $P=.819$ ), and body mass index (mean, $33.56 \pm$ $7.01 \mathrm{~kg} / \mathrm{m}^{2}$ [range, $25.53-48.89 \mathrm{~kg} / \mathrm{m}^{2}$ ], $P=.982$ ). Control subjects were recruited from the obesity center of the University Hospital and from the hospital staff. Exclusion criteria included a known history of central nervous system disease or primary headache syndromes (migraine, tension-type headache, trigeminal neuralgia, cluster headache, hemicrania continua).

\section{MR Imaging}

MR imaging was performed on a 1.5T scanner (Avanto Magnetom; Siemens, Erlangen, Germany). A surface loop coil (medium size, $70 \mathrm{~mm}$ diameter; Siemens) was used in addition to the circularly polarized head coil. The surface coil was placed over the eye with maximum visual disturbances within the head coil and fixed with tape. A coronal non-fat-saturated TSE sequence with a TR of $6960 \mathrm{~ms}$, a TE of $99 \mathrm{~ms}$, an FOV of $85 \times 85 \mathrm{~mm}^{2}$, a matrix size of $256 \times 256 \mathrm{~mm}^{2}$ (in-plane resolution, $0.332 \times 0.332 \mathrm{~mm}^{2}$ ), and contiguous sections with a section thickness of $2 \mathrm{~mm}$ was used to evaluate the $\mathrm{ON}$ and ONS. The acquisition time was 7 minutes 20 seconds. A sagittal non-fat-saturated T2-weighted TSE sequence with a TR of $5170 \mathrm{~ms}$, a TE of $99 \mathrm{~ms}$, an FOV of $170 \times 170 \mathrm{~mm}^{2}$, a matrix size of $256 \times 320 \mathrm{~mm}^{2}$ (in-plane resolution, $0.664 \times 0.531$ $\mathrm{mm}^{2}$ ), and contiguous sections with a section thickness of $2 \mathrm{~mm}$ was used to evaluate the hypophysis. The acquisition time was 5 minutes 27 seconds. Additionally, MR imaging of the whole brain, including a time-of-flight venography (TR, $23 \mathrm{~ms}$; TE, $6.5 \mathrm{~ms}$; FOV, $250 \times 250$ $\mathrm{mm}^{2}$; section thickness, $2 \mathrm{~mm}$; acquisition time, 7 minutes $40 \mathrm{sec}-$ onds) was performed to exclude intracranial pathology and venous sinus thrombosis as a secondary cause of IIH and to identify venous sinus stenoses.

\section{Volume Segmentation}

MR images were converted from DICOM into Neuroimaging Informatics Technology Initiative format by using the DICOM to Neuroimaging Informatics Technology Initiative format converter dcm2nii (http://www.mccauslandcenter.sc.edu/mricro/ mricron/dcm2nii.html). Volume measurements were performed by using Amira 5.3.2 (Visage Imaging, San Diego, California) on a Debian Linux distribution. On each T2-weighted coronal image, the ON and ONS were marked by a region of interest, starting from the papilla and ending at the point of convergence of the extraocular muscles at the tendinous ring of the orbital apex. The mean number of sections was 12 with a section thickness of $2 \mathrm{~mm}$ (Fig 3 and On-line Fig 1). On each T2-weighted sagittal image, the hypophysis was marked with a region of interest. The mean number of sections was 4, with a section thickness of $2 \mathrm{~mm}$ (Fig 3 and On-line Fig 1). Neuroradiologists were blinded with respect to the subject group (patients with IIH versus healthy volunteers), and the interobserver variability was calculated. 

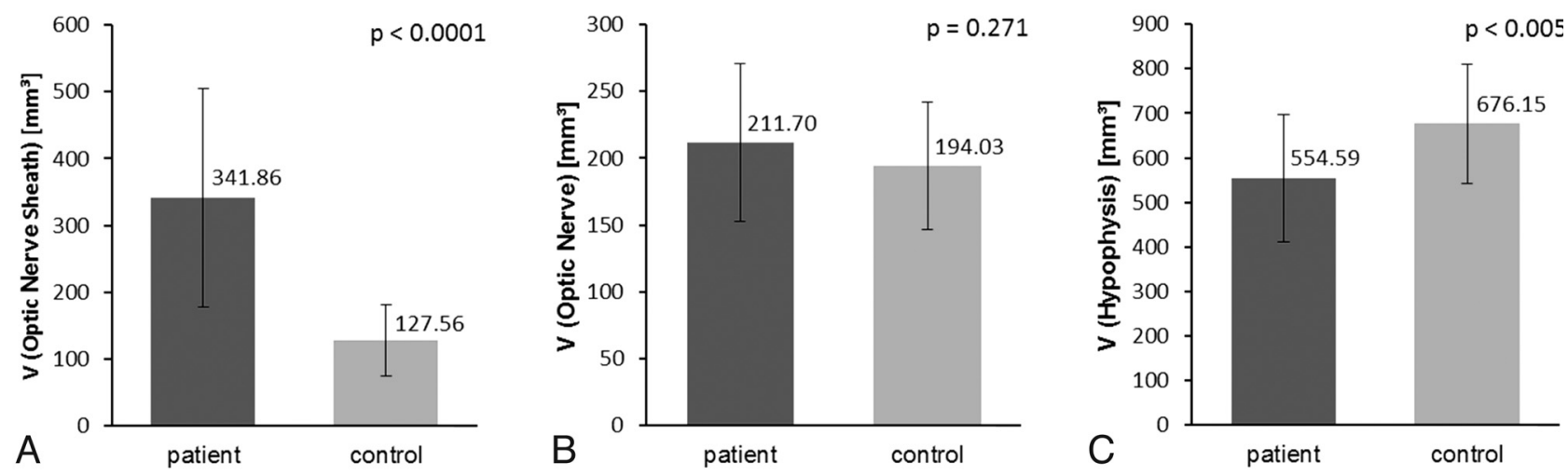

FIG 1. Significance of measurements. A, Significance of ONS volume. Bar graph shows significant differences of mean ONS volumes and corresponding ranges of patient and control groups. B. Significance of OS volume. Note a tendency toward an increased mean OS volume in patients with IIH compared with controls, which, however, does not reach statistical significance. C, Significance of hypophysis volume. This bar graph clearly shows significantly lower mean volumes of hypophysis in patients compared with controls.

\section{Morphologic Analysis}

The configuration of the hypophysis was classified into 4 categories according to a modification of the hypophysis/sella turcica ratio of Yuh et $\mathrm{al}^{10}$ : convex, mild concave (more than one-half the height of the sella), severe concave (less than one-half the height of the sella), and empty sella ( $\leq 2 \mathrm{~mm})$. The appearance of the superior boarder of the hypophysis was classified into anterior and posterior or equal (without predominance of either anterior or posterior deviation of the hypophysary stalk) formation relative to the direction of displacement of the hypophysary stalk.

The classification of stenoses was defined by the highest percentage of stenosis on axial images and maximum-intensity-projection images. Stenoses were categorized into either stenosis of $<50 \%$ or $>50 \%$ of the lumen. Absence of any discontinuity was classified as nonoccurrence of stenosis. Venous sinus stenoses were classified into a left and/or right position as well as assigned to the transverse or sigmoid sinus. The incidence of bilateral stenoses (stenoses in left and right transverse or sigmoid sinus) was statistically listed as 2 stenoses.

\section{Statistical Analysis}

The volumetric data were analyzed by using XLSTAT, Version 2011.3.01 (Addinsoft SARL, New York, New York). The Student $t$ test was used to compare the group means. Statistical significance was assumed at $P<.05$. The Pearson correlation coefficient and correlation matrices were calculated to identify the strength of the correlation of the different parameters. Receiver operating characteristic analysis was used to select an optimal cut-point for prediction and to assess the predictive value in terms of sensitivity, specificity, and accuracy. ${ }^{11}$ Selection of the optimal cut-point was based on the Youden index (ie, the maximum sum of sensitivity and specificity). The 95\% confidence interval for the area under curve was calculated by bootstrapping.

Interobserver variability was determined by calculating intraclass correlation coefficients, including $95 \%$ confidence intervals, for volumetric measurements of ON, ONS, and hypophysis volumes by using PASW Statistics 18, release Version 18.0.0 (IBM, Armonk, New York).

\section{RESULTS}

\section{Clinical Profile of Patients with IIH}

Sixteen $(69.6 \%)$ patients presented with headache. The mean pain intensity was $2.22 \pm 2.53$ (range, $0.0-7.5$ ) according to the visual analog scale before examination, and a mean pain intensity maximum was $7.09 \pm 2.62$ (range, 0.95-9.86) (visual analog scale). Impaired vision was reported by $91.30 \%$ of patients with IIH. The visual impairment was categorized according to the On-line Table: worsening of visual acuity, $47.83 \%$; flashes, $39.13 \%$; transient visual obscurations, $43.48 \%$; scotoma, $73.91 \%$; papilledema, $56.52 \%$; blurred vision, $52.17 \%$; and diplopia, $43.48 \%$. No case of optic nerve atrophy was observed. Of the $39.13 \%$ of patients reporting tinnitus, $44.44 \%$ characterized their tinnitus as pulsatile. Light sensitivity (60.87\%), noise sensitivity (52.17\%), and vertigo (60.87\%) were common symptoms. Medical history revealed a mean value of maximum CSF opening pressure of $37.61 \pm 6.93$ $\mathrm{cm} \mathrm{H}_{2} \mathrm{O}$ (range, 29.0-50.0 $\mathrm{cm} \mathrm{H}_{2} \mathrm{O}$ ) (On-line Table).

\section{Comparison of Volume Parameters among Patients with IIH and Controls}

Patient mean ONS volume (On-line Fig $1 A$ ) was $341.86 \pm 163.69$ $\mathrm{mm}^{3}$ (range, 105.61-796.57 $\mathrm{mm}^{3}$ ), whereas control mean ONS volume was $127.56 \pm 53.17 \mathrm{~mm}^{3}$ (range, $29.24-239.00 \mathrm{~mm}^{3}$ ) $(P<.0001)$. Mean ON volume for patients (On-line Fig $1 B)$ was $211.70 \pm 59.22 \mathrm{~mm}^{3}$ (range, $125.67-319.00 \mathrm{~mm}^{3}$ ), and for controls, $194.03 \pm 47.77 \mathrm{~mm}^{3}$ (range, $126.56-342.61 \mathrm{~mm}^{3}$ ), with no significant difference $(P=.271)$. Mean hypophysis volume (Online Fig $1 C$ ) of patients with IIH was $554.59 \pm 142.82 \mathrm{~mm}^{3}$ (range, $\left.334.43-855.15 \mathrm{~mm}^{3}\right)$; that of controls was $676.15 \pm 133.32 \mathrm{~mm}^{3}$ (range, 392.86-1045.65 $\left.\mathrm{mm}^{3}\right)(P<.005)$. The intraclass correlation coefficient for ON volume was 0.940 (95\% CI, 0.864-0.974); for ONS volume, it was 0.947 (95\% CI, 0.939-0.989); and for hypophysis volume, 0.981 (95\% CI, 0.957-0.992).

\section{Cutoff Values}

The receiver operating characteristic analysis (Fig 3 and Table) revealed an optimal cutoff value of $201.30 \mathrm{~mm}^{3}$ for mean ONS volume, with a sensitivity of $86.96 \%$, a specificity of $91.30 \%$, and an accuracy of $89.13 \%$ to distinguish those with IIH and healthy controls. For mean hypophysis volumes, the optimal cutoff value 
was $611.21 \mathrm{~mm}^{3}$, with a sensitivity of $78.26 \%$, a specificity of $69.57 \%$, and an accuracy of $73.91 \%$. With area under the curve values of 0.934 and 0.724 , respectively, receiver operating characteristic analysis showed significant group differences for mean ONS volume $(P<.0001)$ and for hypophysis volume $(P<.005)$. For mean $\mathrm{ON}$ volume, the receiver operating characteristic analysis showed no significant difference between the groups; the area under curve was $0.580(P=.338)$.

\section{Morphology of the Hypophysis}

Morphology of the hypophysis in patients with IIH showed 0 convex, 4 mild concave, 11 severe concave, and 8 empty sella configurations, whereas controls had 13 convex $(P<.05)$, 4 mild

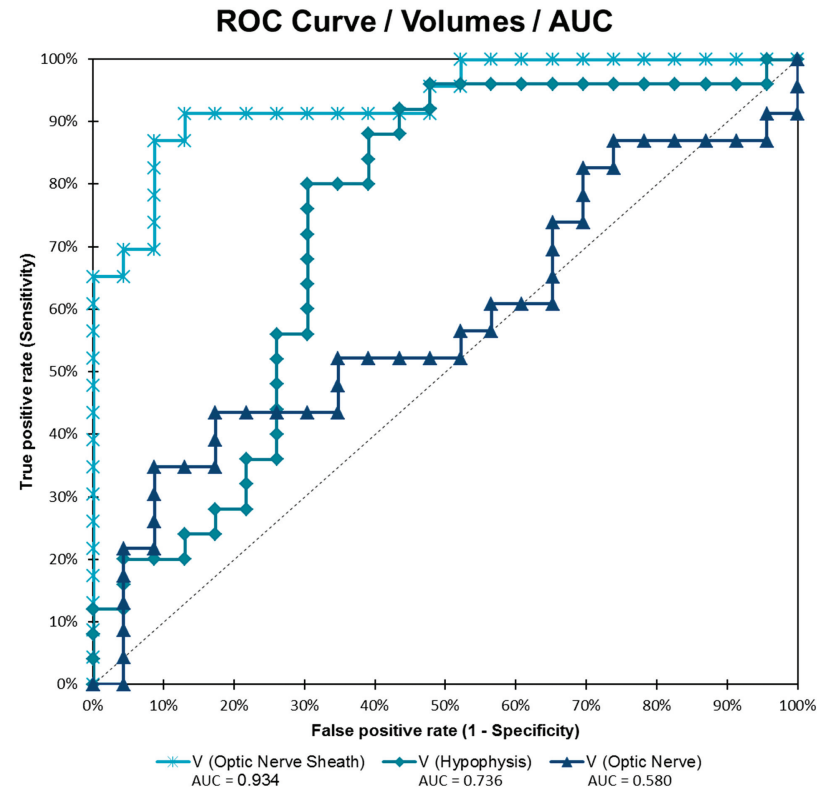

FIG 2. Receiver operating characteristic analysis curve for volumes of ONS, ON, and hypophysis.
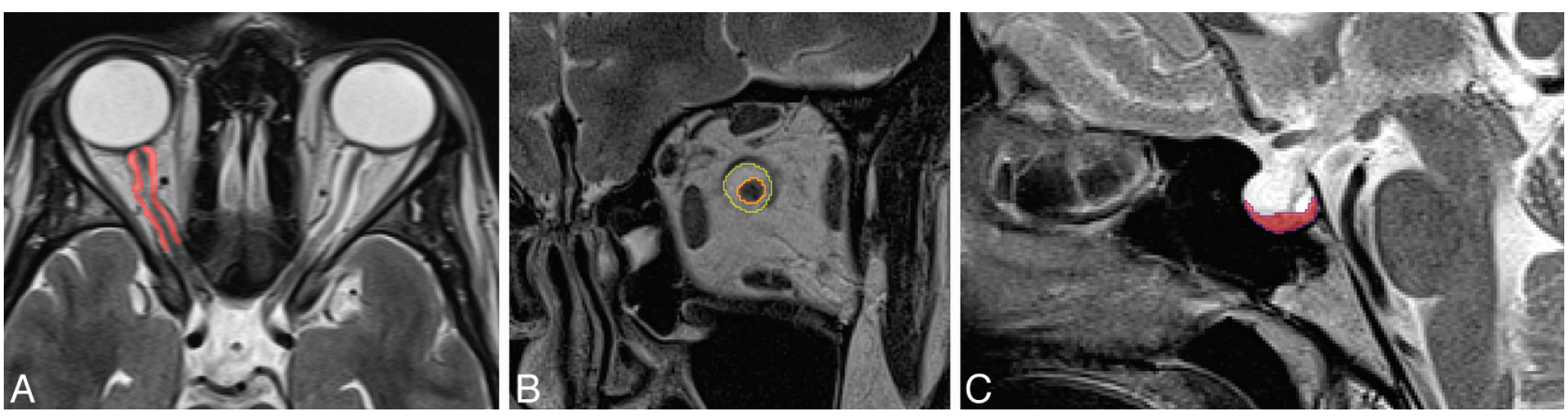

FIG 3. Voxel-based analysis of control MR images of healthy controls and normal configuration of the ONS and hypophysis. A, Axial scan with segmentation of ONS volume (red). B, Coronal scan with selection of ONS (yellow) and ON (red) volume segmentation. C, Midsagittal scan shows normal configuration of the hypophysis.

Area under the receiver operating characteristic curve and cut-off values

\begin{tabular}{lcccccc} 
& & & & \multicolumn{2}{c}{$95 \% \mathrm{Cl}$} & \\
\cline { 5 - 6 } Parameter (Volume) & AUC & SE & $\boldsymbol{P}$ & Lower Bound & Upper Bound & Cut-Off Value ${ }^{\mathrm{a}}$ (mm) \\
\hline ONS & 0.934 & 0.027 & $<.0001$ & 0.881 & 0.987 & 201.30 \\
ON & 0.580 & 0.084 & .338 & 0.416 & 0.745 & 228.64 \\
Hypophysis & 0.724 & 0.076 & .003 & 0.574 & 0.874 & 611.21 \\
\hline
\end{tabular}

Note:-AUC indicates area under the curve.

${ }^{a}$ Calculated with the Youden Index ( $Y=$ Sensitivity + Specificity-1). 
the radiologic diagnosis is critically affected by the experience of the radiologist.

Several recent studies have reported significant correlations of imaging findings, such as hypophysis compression and empty sella, ONS distension, and ON tortuosity, with the diagnosis of IIH. ${ }^{3,6,12}$ Measurements of ONS diameters demonstrated wider ONS in individuals with IIH. ${ }^{6,13}$ On the other hand, a few studies have questioned these findings as not being statistically significant. $^{6,14,15}$ Morphologic studies of the hypophysis in IIH were more consistent and showed a significant reduction of the ratio of the hypophysis to the sella turcica in patients with IIH. ${ }^{10,14}$ Relevance of slit-like ventricular morphology ${ }^{16}$ was postulated in early reports, but subsequent studies dissented from this hypothesis by verifying normal ventricle configuration in patients with $\mathrm{IIH} .{ }^{17}$

We have shown that volumetric measurements of ONS and hypophysis volumes can be used to accurately diagnose patients with IIH. ONS volumes of $>201.30 \mathrm{~mm}^{3}$ (sensitivity, $86.96 \%$; specificity, 91.30\%; accuracy, 89.13\%) and hypophysis volumes of $<611.21 \mathrm{~mm}^{3}$ (sensitivity, 78.26\%; specificity, $69.57 \%$; accuracy, $73.91 \%$ ) were significantly associated with the diagnosis of IIH. In comparison with previous studies by using metric analysis of ONS (sensitivity, 66.7\%; specificity, 82.1\%; accuracy, 76.73\%) and hypophysis (sensitivity, 53.3\%; specificity, 75\%; accuracy, $67.44 \%$ ), volumetric measurements increase the diagnostic reliability, ${ }^{3}$ including low interobserver variability. Brodsky ${ }^{6}$ observed an increased ONS diameter in 9 of 20 patients (45\%), whereas our findings showed a dilated perioptic subarachnoid space in 20 of 23 patients (86.96\%). Among patients with IIH, we did not observe a significant correlation between imaging findings and CSF opening pressure (ONS: $\mathrm{cc}=0.0307, P=.892$; hypophysis: $c c=0.2055, P=.359)$. Therefore, the results suggest that abnormal imaging findings may not be exclusively a direct result of elevated CSF pressure. Consequently, definite diagnosis (ie, according to the revised Dandy criteria) still requires liquor puncture (intracranial pressure $>25 \mathrm{~cm} \mathrm{H}_{2} \mathrm{O}$ ). ${ }^{9}$

The underlying cause of IIH still remains largely unknown, but the hypotheses of increased CSF production, reduced CSF absorption, and venous outflow obstruction as well as metabolic alterations are discussed. ${ }^{18,19}$ Venous sinus stenoses may lead to increased resistance in venous drainage and, as a consequence, to increased intracranial pressure. It is controversially debated whether obstructed venous drainage is the primary mechanism or is secondary to another pathologic process. In this context, compressed venous sinuses may also be the result of external compression $^{20-24}$ by increased intracranial pressure. Galgano and Deshaies $^{25}$ stated that flow-related artifacts in noncontrast MR venography may be challenging to distinguish from pathologic venous sinus stenosis. Therefore, venous sinus abnormalities in IIH have to be assessed carefully. ${ }^{25,26}$ We did observe venous sinus stenoses in 6 patients with $\mathrm{IIH}$ and in 1 healthy control. In the present study, TOF-MR venography was performed to identify venous sinus abnormalities. However, detection of stenosis definitely depends on the imaging technique used. Compared with contrast-enhanced MRA or DSA and even though analyzed by a highly experienced neuroradiologist, results are slightly more prone to misinterpretation, owing to the underlying technical limitations of TOF-MR venography. ${ }^{27,28}$
In our study, the mean $\mathrm{ON}$ volume of patients was slightly increased compared with that in controls $\left(211.7 \mathrm{~mm}^{3}\right.$ versus $194.03 \mathrm{~mm}^{3}$, On-line Fig $1 B$ ), but this effect was not statistically significant ( $P=.271$; sensitivity, $43.48 \%$; specificity, $82.61 \%$ ). It has been assumed that increased intracranial pressure leads to increased transmission of CSF into the intraorbital ONS, which impedes axoplasmic transport of synaptic vesicles, organelles, and molecules followed by optic nerve fiber swelling and flattening of the posterior globe. ${ }^{29}$ Yet, we have not found a correlation of intracranial pressure and volume parameters supporting this hypothesis. Neither the degree of hypophysis compression nor the distention of ONS was augmented with increasing intracranial pressure. These results are in agreement with the observation that reduction of intracranial pressure by lumbar puncture has only a temporary effect. ${ }^{30}$ Furthermore, these findings indicate that macroscopic deformations are indirect or comorbid changes rather than direct results of increased intracranial pressure. Therefore, it remains unclear in which way and to what extent the CSF space is affected in response to increasing intracranial pressure. We have considered the body mass index, which is especially important due to the strong association between IIH and obesity. ${ }^{18,31-35}$

Morphologic signs become clearly visible only in advanced disease stages on conventional MR imaging analysis, whereas volumetric measurements allow an improved diagnostic reliability and possibly earlier detection, with potential impact on monitoring treatment efficacy. In a routine radiologic setting, image postprocessing with quantitative analysis as previously specified can be achieved rapidly ( $\leq 10$ minutes) by experienced radiologists. Software-assisted volumetric measurement has the potential to facilitate decision-making due to objective statistical parameters.

Possible automatization of measurement procedures could provide even faster and more reliable ways of assessment. ${ }^{36,37}$ Our relatively small study sample represents a potential limitation and requires confirmation by subsequent investigations.

\section{CONCLUSIONS}

As opposed to MR imaging-based metric measurements of hypophysis and ONS in patients with IIH, which have a confined diagnostic value due to their limited reproducibility and strong observer dependency, semiautomated volumetric analysis has the potential to objectify diagnosis and follow-up procedures by reducing interobserver bias.

Disclosures: Jan Hoffmann—UNRELATED: Grants/Grants Pending: German Research Foundation (Deutsche Forschungsgemeinschaft), Travel/Accommodations/ Meeting Expenses Unrelated to Activities Listed: Allergan (travel expenses to meeting). Hagen Kunte-UNRELATED: Payment for Lectures (including service on Speakers Bureaus): Biogen-Idec (for speaking), Travel/Accommodations/Meeting Expenses Unrelated to Activities Listed: Biogen-Idec, Merck-Serono, Almirall. Lutz Harms-UNRELATED: Board Membership: Biogen Idec, Sanofi, Novartis, Consultancy: Biomarin, Payment for Lectures (including service on Speakers Bureaus): Biogen Idec, Bayer, Merck Serono, Novartis; Travel/Accommodations/Meeting Expenses Unrelated to Activities Listed: Bayer, Biogen Idec. Lutz Lüdemann-UNRELATED: Consultancy: local state ethics committee of the city of Berlin, Grants/ Grants Pending: Deutsche Forschungsgemeinschaft, German research community, ${ }^{*}$ Comments: Project: biological radiation treatment planning. Payment for Lectures (including service on Speakers Bureaus): Haus der Technik Essen. *Money paid to the institution.

AJNR Am J Neuroradiol 35:513-18 Mar 2014 www.ajnr.org 


\section{REFERENCES}

1. Pearce JM. From pseudotumour cerebri to idiopathic intracranial hypertension. Pract Neurol 2009;9:353-56

2. Spennato P, Ruggiero C, Parlato RS, et al. Pseudotumor cerebri. Childs Nerv Syst 2011;27:215-35

3. Agid R, Farb RI, Willinsky RA, et al. Idiopathic intracranial hypertension: the validity of cross-sectional neuroimaging signs. Neuroradiology 2006;48:521-27

4. Friedman DI, Jacobson DM. Diagnostic criteria for idiopathic intracranial hypertension. Neurology 2002;59:1492-95

5. Skau M, Brennum J, Gjerris F, et al. What is new about idiopathic intracranial hypertension? An updated review of mechanism and treatment. Cephalalgia 2006;26:384-99

6. Brodsky M. Magnetic resonance imaging in pseudotumor cerebri. Ophthalmology 1998;105:1686-93

7. Headache Classification Subcommitte of the International Headache Society. The International Classification of Headache Disorders. 2nd edition. Cephalalgia 2004;24(suppl 1):9-160

8. World Health Organization. International Statistical Classification of Diseases and Related Health Problems. Geneva, Switzerland: World Health Organization; 2004

9. Friedman DI. Idiopathic intracranial hypertension with Dan and beyond: the 2010 Jacobson Lecture. J Neuroophthalmol 2010; 30:380-85

10. Yuh WT, Zhu M, Taoka T, et al. MR imaging of pituitary morphology in idiopathic intracranial hypertension. J Magn Reson Imaging 2000;12:808-13

11. Bewick V, Cheek L, Ball J. Statistics review 13: receiver operating characteristic curves. Crit Care 2004;8:508-12

12. Manfré L, Lagalla R, Mangiameli A, et al. Idiopathic intracranial hypertension: orbital MRI. Neuroradiology 1995;37:459-61

13. Degnan AJ, Levy LM. Narrowing of Meckel's cave and cavernous sinus and enlargement of the optic nerve sheath in pseudotumor cerebri. J Comput Assist Tomogr 2011;35:308-12

14. Gibby WA, Cohen MS, Goldberg HI, et al. Pseudotumor cerebri: CT findings and correlation with vision loss. $A J R A m$ J Roentgenol 1993;160:143-46

15. Gass A, Barker GJ, Riordan-Eva P, et al. MRI of the optic nerve in benign intracranial hypertension. Neuroradiology 1996;38:769-73

16. Weisberg LA. Computed tomography in benign intracranial hypertension. Neurology 1985;35:1075-78

17. Jacobson DM, Karanjia PN, Olson KA, et al. Computed tomography ventricular size has no predictive value in diagnosing pseudotumor cerebri. Neurology 1990;40:1454-55

18. Kesler A, Kliper E, Shenkerman G, et al. Idiopathic intracranial hypertension is associated with lower body adiposity. Ophthalmology 2010;117:169-74

19. Degnan AJ, Levy LM. Pseudotumor cerebri: brief review of clinical syndrome and imaging findings. AJNR Am J Neuroradiol 2011;32:1986-93
20. Ahmed R, Friedman DI, Halmagyi GM. Stenting of the transverse sinuses in idiopathic intracranial hypertension. J Neuroophthalmol 2011;31:374-80

21. Stienen A, Weinzierl M, Ludolph A, et al. Obstruction of cerebral venous sinus secondary to idiopathic intracranial hypertension. Eur J Neurol 2008;15:1416-18

22. Higgins JN, Pickard JD. Lateral sinus stenoses in idiopathic intracranial hypertension resolving after CSF diversion. Neurology 2004;62:1907-08

23. Lee SW, Gates $\mathrm{P}$, Morris $\mathrm{P}$, et al. Idiopathic intracranial hypertension; immediate resolution of venous sinus "obstruction" after reducing cerebrospinal fluid pressure to $<10 \mathrm{cmH}(2) \mathrm{O}$. J Clin Neurosci 2009;16:1690-92

24. Scoffings DJ, Pickard JD, Higgins JN. Resolution of transverse sinus stenoses immediately after CSF withdrawal in idiopathic intracranial hypertension. J Neurol Neurosurg Psychiatry 2007;78:911-12

25. Galgano MA, Deshaies EM. An update on the management of pseudotumor cerebri. Clin Neurol Neurosurg 2013;115:252-59

26. Peng KP, Fuh JL, Wang SJ. High-pressure headaches: idiopathic intracranial hypertension and its mimics. Nat Rev Neurol 2012;8:700-10

27. Nedelmann M, Kaps M, Mueller-Forell W. Venous obstruction and jugular valve insufficiency in idiopathic intracranial hypertension. J Neurol 2009;256:964-69

28. Fera F, Bono F, Messina D, et al. Comparison of different MR venography techniques for detecting transverse sinus stenosis in idiopathic intracranial hypertension. J Neurol 2005;252:1021-25

29. Acheson JF. Idiopathic intracranial hypertension and visual function. Br Med Bull 2006;79-80:233-44

30. Johnston I, Paterson A. Benign intracranial hypertension. II. CSF pressure and circulation. Brain 1974;97:301-12

31. Kupersmith MJ, Gamell L, Turbin R, et al. Effects of weight loss on the course of idiopathic intracranial hypertension in women. $\mathrm{Neu}$ rology 1998;50:1094-98

32. Wall M. Idiopathic intracranial hypertension. Neurol Clin 2010;28:593-617

33. Ko MW, Chang SC, Ridha MA, et al. Weight gain and recurrence in idiopathic intracranial hypertension: a case-control study. Neurology 2011;76:1564-67

34. Ball AK, Clarke CE. Idiopathic intracranial hypertension. Lancet Neurol 2006;5:433-42

35. Sugerman HJ, Felton WL 3rd, Salvant JB Jr, et al. Effects of surgically induced weight loss on idiopathic intracranial hypertension in morbid obesity. Neurology 1995;45:1655-59

36. Kassubek J, Pinkhardt EH, Dietmaier A, et al. Fully automated atlasbased MR imaging volumetry in Huntington disease, compared with manual volumetry. AJNR Am J Neuroradiol 2011; 32:1328-32

37. Huppertz HJ, Kroll-Seger J, Danek A, et al. Automatic striatal volumetry allows for identification of patients with chorea-acanthocytosis at single subject level. J Neural Transm 2008;115:1393-400 